\title{
Effect of Cross-linker Multiplicity on the Gel Point in ATRP
}

\author{
Wim Van Camp ${ }^{1,2}$, Haifeng Gao ${ }^{2}$, Filip E. Du Prez ${ }^{1}$, Krzysztof Matyjaszewski ${ }^{1, *}$
}

${ }^{1}$ Center for Macromolecular Engineering, Department of Chemistry, Carnegie Mellon

University, 4400 Fifth Avenue, Pittsburgh, Pennsylvania 15213

${ }^{2}$ Polymer Chemistry Research Group, Department of Organic Chemistry, Ghent University, Krijgslaan 281 S4, B-9000 Ghent, Belgium

*Corresponding author. Email: km3b@andrew.cmu.edu

\section{Keywords}

Gel, ATRP, cross-linker, multiplicity, branching 


\section{Abstract}

The experimental gelation was studied in atom transfer radical polymerization (ATRP) of methyl acrylate (MA) with various branching reagents that structurally differ by the number of vinyl groups (multiplicity). MA was copolymerized with branching reagents containing 2, 3, 4, 5 or 6 acrylate moieties per molecule, respectively. Reactions with a constant concentration of branching vinyl groups (the same molar ratio of [Vinyl $\left.\mathrm{branch}_{0}\right]_{0} /[\text { Initiator }]_{0}$ ) revealed a different gelation behavior when ethylene glycol diacrylate (2A) and trimethylolpropane triacrylate (3A) were used as cross-linkers, while the reactions using pentaerythritol tetraacrylate (4A) showed similar gelation behavior as compared to reactions using 3A. Additional reactions with dipentaerythritol pentaacrylate (5A) and hexafunctional acrylate cross-linkers (6A) revealed the presence of a concentration-dependant gelation behavior. On the other hand, in reactions with the same molar concentration of various crosslinkers, gelation occurred at progressively lower MA conversions for reactions with an increasing number of vinyl groups per cross-linker. In addition, the number of unreacted pendant vinyl groups in the sols was compared for reactions with different multiplicity of the cross-linker and various ratios of $\left[\mathrm{Viny}_{\mathrm{branch}}\right]_{0} /[\text { Initiator }]_{0}$. Finally, a linear oligomeric crosslinker containing multiple branching vinyl groups along the side chain was used as the branching reagent for gelation. The gelation behavior during the ATRP of MA with the linear cross-linker was different as compared to the use of the above-mentioned cross-linkers with a star-like architecture. 


\section{Introduction}

Conventional radical polymerization (RP) of a monovinyl monomer with a small amount of divinyl cross-linker is a widespread method for the synthesis of branched polymers or gels. However, the slow and continuous initiation process, fast chain propagation and termination reactions that are inherent to conventional RP result in networks with an inhomogeneous and uncontrolled structure. ${ }^{1-4}$ Recently, several research groups have shown that the application of controlled radical polymerization (CRP) techniques ${ }^{5-9}$ produces gels with a more homogeneous structure, as a result of the fast initiation process and reversible deactivation of propagating polymer chains. ${ }^{10-13}$ The fast initiation reactions, relative to propagation, result in a quick conversion of nearly all initiators to growing primary chains. On the other hand, the dynamic equilibrium between the active species, bearing a radical at the polymer chain end, and the dormant species ensures a low steady concentration of radicals. During each activation/deactivation cycle, only a few monomers are added to the growing polymer chain before it is quickly deactivated to the dormant state. The dormant state allows for chain relaxation and diffusion of reagents. As a result, the probability of reaction of each vinyl species, monomer, 'unreacted' cross-linker or pendant vinyl group, is dependent on their relative concentration and reactivity. Therefore, the branched polymers or gels have a more homogeneous distribution of branching points than the polymers synthesized by RP methods at similar reaction conditions. ${ }^{11}$

Recently, several research groups systematically studied the synthesis of branched polymers and/or gels by the copolymerization of monovinyl monomer and divinyl cross-linker using atom transfer radical polymerization (ATRP). ${ }^{10,14-25}$ Sherrington $^{20,21}$ and Armes ${ }^{18,19,22}$ focused on the synthesis of branched polymers based on copolymerization of methacrylate and dimethacrylate cross-linker. Zhu et al. studied the homopolymerization ${ }^{25}$ of ethylene glycol dimethacrylate and copolymerization ${ }^{23,24}$ of methyl methacrylate and dimethacrylate cross- 
linker using bulk ATRP. Our group applied the ATRP of methyl acrylate (MA) and ethylene glycol diacrylate as a model system to study the dependence of the experimental gel points on various parameters, because of its fast initiation process, high initiation efficiency, and low polydispersity in all performed experiments. ${ }^{14}$ The effect of several parameters was studied, e.g. the molar ratio of divinyl cross-linker to initiator, ${ }^{14}$ the concentration of all reagents during the gelation process, ${ }^{15}$ the relative reactivity of divinyl cross-linker compared to monomer, ${ }^{16}$ and the initiation efficiency and polydispersity of the primary chains. ${ }^{17}$ Furthermore, the experimental results were compared with values obtained from Monte Carlo simulations. $^{26}$ Finally, an $\mathrm{AB}^{*}$ inimer (initiator-monomer containing vinyl bond $\mathrm{A}$ and initiator fragment $\mathrm{B}^{*}$ in one molecule) was also explored as a branching reagent for the synthesis of branched copolymers and gels by copolymerization with monomer and divinyl cross-linker using ATRP, ${ }^{27}$ and the results were compared for gelation reactions using divinyl and trivinyl cross-linker.

In this paper, we aim to expand the systematic study on the gelation in ATRP and investigate the effect of cross-linker multiplicity, i.e. the number of vinyl groups per cross-linker molecule, on the experimental gel points. Copolymerization of MA was carried out with various branching reagents: ethylene glycol diacrylate (2A), trimethylolpropane triacrylate (3A), pentaerythritol tetraacrylate (4A), dipentaerythritol pentaacrylate (5A) and a hexafunctional acrylate (6A) cross-linker (see Scheme 1 and Scheme 2). In order to keep the system comparable to those previously studied, the experimental gel points based on the monomer (MA) conversions were determined and systematically compared to each other for reactions where the molar ratios of branching vinyl groups to initiator ([Vinyl $\mathrm{branch}_{0} /[\text { Initiator }]_{0}$ ) and the cross-linker species were varied. The effect of cross-linker multiplicity was also investigated in a series of reactions where the molar concentration of the various cross-linkers was kept constant. Additionally, the behavior of the above-mentioned 
star-like cross-linkers (3A, 4A, 5A, 6A) was compared with a linear oligomeric multivinyl cross-linker molecule, containing an average of 7 dangling vinyl groups along the polymer chain.

Scheme 1. Synthesis of polymer networks by ATRP of MA and different branching reagents. 

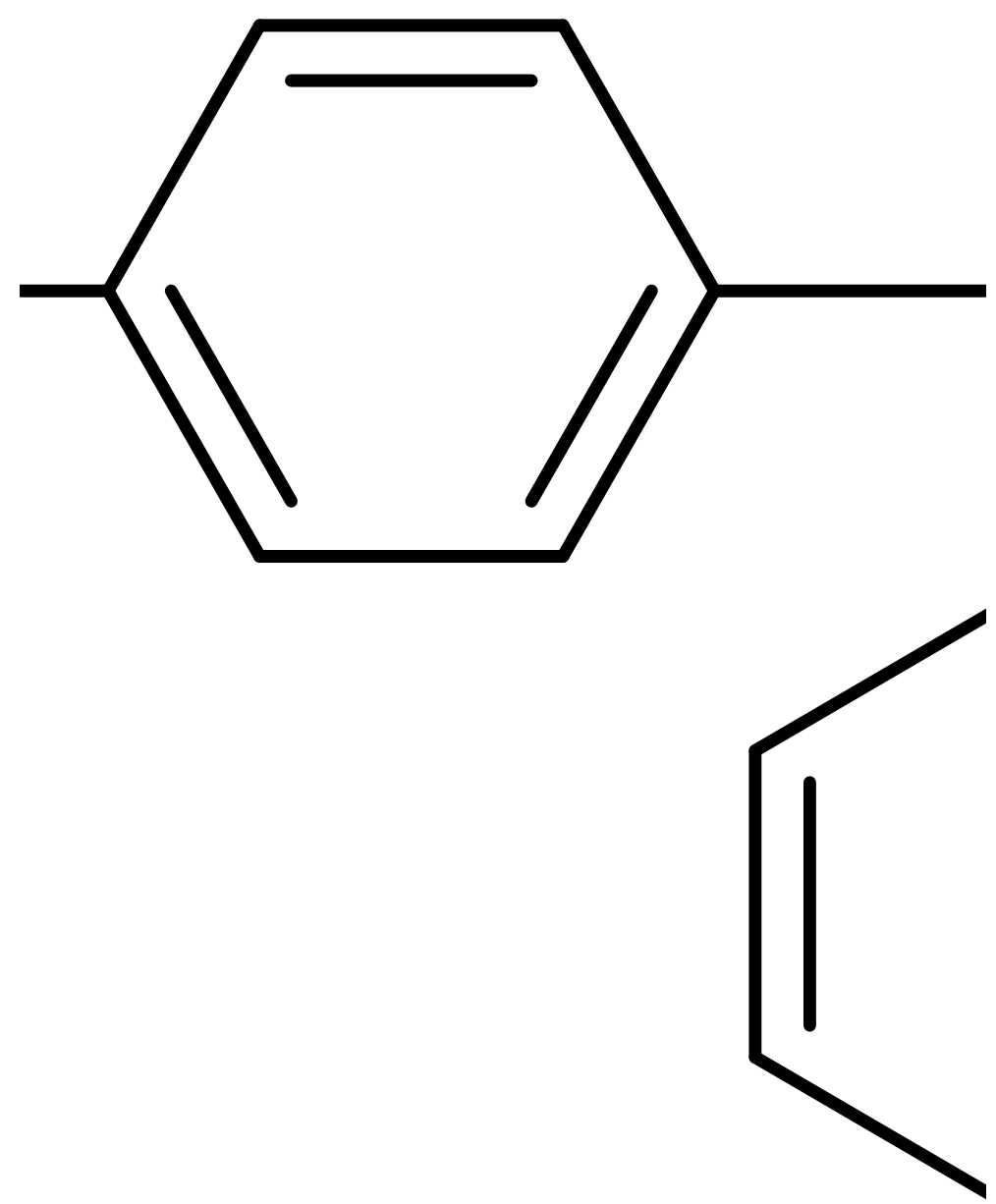

Scheme 2. Synthesis of hexafunctional acrylate cross-linker (6A).

\section{Experimental Section}

Materials. Methyl acrylate (MA, 99\%), 2-hydroxyethyl acrylate (HEA, 96\%), n-butyl acrylate (nBA, 99\%) and ethylene glycol diacrylate (2A, 90\%) were purchased from Aldrich 
and purified twice by passing through a column filled with basic alumina to remove the inhibitor. The trivinyl cross-linker, trimethylolpropane triacrylate (3A, technical grade, Aldrich) and tetravinyl cross-linker, pentaerythritol tetraacrylate (4A, technical grade, Aldrich) was purified via silica liquid chromatography with mixture of hexane/ethyl acetate 7/3 by volume as the eluent. Dipentaerythritol penta-/hexa-acrylate (5A, technical grade, Aldrich) was determined to have an average multiplicity of 5 by ${ }^{1} \mathrm{H}$ NMR, and was used as received. $\mathrm{CuBr}$ (98\%, Acros) was purified using a modified literature procedure. ${ }^{28}$ All other reagents: methyl 2-bromopropionate (MBP), ethyl 2-bromopropionate (EBrP), $N, N, N$ ', $N$ ", $N$ ”-pentamethyldiethylenetriamine (PMDETA), acryloyl chloride, $\quad N, N$ 'dicyclohexylcarbodiimide (DCC), 4-(dimethylamino)pyridine, p-toluenesulfonic acid monohydrate, 2,2-bis(hydroxymethyl)propionic acid, 2,2-dimethoxypropane, triethyl amine, 1,1,1-tris(4-hydroxyphenyl)ethane, 4-Hydroxy-TEMPO, $\mathrm{CuBr}_{2}$ and solvents were purchased from Aldrich with the highest purity and used as received without further purification.

Characterization. Monomer conversions were determined from the concentration of the unreacted monomer in the samples periodically removed from the reactions using a Shimadzu GC-17A gas chromatograph, equipped with a capillary column (DB-Wax, $30 \mathrm{~m} \times$ $0.54 \mathrm{~mm} \times 0.5 \mu \mathrm{m}, \mathrm{J} \& \mathrm{~W}$ Scientific). DMF was used as internal standard for calculation of monomer conversions. After filtration through 220 nm PTFE filter, the polymer samples were separated by GPC (Polymer Standards Services (PSS) columns (guard, $10^{5}, 10^{3}$, and $10^{2} \AA$ ), with THF eluent at $35{ }^{\circ} \mathrm{C}$, flow rate $=1.00 \mathrm{~mL} / \mathrm{min}$ and differential refractive index (RI) detector (Waters, 2410)). The apparent molecular weights and polydispersities $\left(\mathrm{M}_{\mathrm{w}} / \mathrm{M}_{\mathrm{n}}\right)$ were determined with a calibration based on linear poly(methyl methacrylate) (polyMMA) standards using WinGPC 6.0 software from PSS. The GPC curves obtained with flat baseline were imported into the WinGPC software for calculation of their apparent molecular weights 
and polydispersity. ${ }^{1} \mathrm{H}$ NMR spectra, using $\mathrm{CDCl}_{3}$ or acetone- $\mathrm{d}_{6}$ as solvent, were measured on a Bruker Avance $300 \mathrm{MHz}$ spectrometer at $27^{\circ} \mathrm{C}$.

\section{Synthesis of hexafunctional acrylate (6A) cross-linker.}

1) Synthesis of 2,2-Bis((2,2-propyl)dioxymethyl)propionic acid $\mathbf{1}$. 2,2bis(hydroxymethyl)propionic acid (bis-MPA) (10.0 g, $74.6 \mathrm{mmol}$ ), was reacted with 2,2dimethoxypropane (13.8 mL, $112 \mathrm{mmol}, 1.5$ eq. to bis-MPA), and p-TSA (0.71 g, 5 mol\% to bis-MPA) in $50 \mathrm{~mL}$ of acetone at room temperature. After $2 \mathrm{~h}, 1 \mathrm{~mL}$ of $\mathrm{NH}_{4} \mathrm{OH}$ (aqueous, $30 \%) / \mathrm{EtOH}(1 / 1)$ solution was added to neutralize the catalyst. The reaction mixture was concentrated by rotary evaporation, the residue was redissolved in $\mathrm{CH}_{2} \mathrm{Cl}_{2}$, and extracted twice with $20 \mathrm{~mL}$ of $\mathrm{H}_{2} \mathrm{O}$. The organic phase was dried over $\mathrm{MgSO}_{4}$, filtered and evaporated to yield $8.3 \mathrm{~g}(63 \%)$ of white crystals. ${ }^{1} \mathrm{H}$ NMR $\left(\mathrm{CDCl}_{3}\right): \delta=1.20$ (s, 3H, $\left.-\mathrm{CH}_{3}\right), 1.41-1.44$ (d, $\left.6 \mathrm{H},-\mathrm{C}\left(\mathrm{CH}_{3}\right)_{2}\right), 3.65-4.20\left(\mathrm{dd}, 4 \mathrm{H},-\mathrm{C}\left(\mathrm{CH}_{2} \mathrm{O}^{-}\right)_{2}\right)$.

2) Synthesis and deprotection of 2. 1,1,1-tris(4-hydroxyphenyl)ethane (3.73 g, 12.2 mmol) and 1 (7.0 g, $40.2 \mathrm{mmol}, 1.1$ eq. to $\mathrm{OH}$ groups) was dissolved in $25 \mathrm{~mL}$ of dry $\mathrm{CH}_{2} \mathrm{Cl}_{2}$. $N, N$ '-dicyclohexylcarbodiimide (DCC) (11.3 g, 1.5 eq. to $\mathrm{OH}$ groups) and 4(dimethylamino)pyridine (1.13 g, $10 \mathrm{w} \%$ to DCC) was added. The mixture was stirred overnight at room temperature. The formed urea was removed by filtration. The filtrate was concentrated and the resulting viscous oil was purified by silica gel column chromatography (eluens hexane/ethyl acetate 3/2). The pure fractions were concentrated, resulting in a viscous oil. After purification, the product was dissolved in a mixture of $30 \mathrm{~mL}$ of THF and $30 \mathrm{~mL}$ of $1 \mathrm{M} \mathrm{HCl}$ (aq.). The reaction mixture was stirred for $2 \mathrm{~h}$ before the precipitated product was filtered and washed with water. The product was dried in vacuum at $50{ }^{\circ} \mathrm{C}$, yielding a white solid. Yield: 3.83 g (90\%). ${ }^{1} \mathrm{H}$ NMR (acetone- $\left.d_{6}\right): \delta=1.31$ (s, 9H,-C $\left.H_{3}\right), 2.21$ (s, 3H, $\left.-\mathrm{CH}_{3}\right), 3.76-3.89\left(\mathrm{~m}, 12 \mathrm{H},-\mathrm{C}\left(\mathrm{CH}_{2} \mathrm{O}^{-}\right)_{2}\right), 7.06-7.17$ (m, 12H, $\left.-\mathrm{OPh}-\right)$. 
3) Esterification with acryloyl chloride. 2 (1.52 g, 2.32 mmol, 13.9 mmol OH groups), $25 \mathrm{~mL}$ dry dichloromethane, triethyl amine $(2.81 \mathrm{~g}, 3.88 \mathrm{~mL}, 27.9 \mathrm{mmol})$ and $10.0 \mathrm{mg}$ of radical inhibitor 4-hydroxy-TEMPO were added to a clean, dry round-bottom flask. The flask was placed in a an ice-water bath and the mixture was cooled down to $0{ }^{\circ} \mathrm{C}$. Acryloyl chloride ( $2.52 \mathrm{~g}, 2.27 \mathrm{~mL}, 27.8 \mathrm{mmol}, 2$ eq. to $\mathrm{OH}$ groups) was added dropwise to the reaction mixture over a period of $20 \mathrm{~min}$. After $1 \mathrm{~h}$, the flask was removed from the ice-water bath and the reaction mixture was stirred for $24 \mathrm{~h}$ at room temperature. During this period, the reaction mixture changed from a yellowish color to brown. The reaction mixture was washed successively with $100 \mathrm{~mL}$ of $1 \mathrm{M} \mathrm{HCl}, 100 \mathrm{~mL}$ of $1 \mathrm{M} \mathrm{NaOH}$, and $100 \mathrm{~mL}$ of deionized water and then dried over anhydrous $\mathrm{MgSO}_{4}$ for $1 \mathrm{~h} .10 \mathrm{mg}$ of radical inhibitor 4-hydroxy-TEMPO was added to the mixture and the solvent was removed via rotary evaporation and oil pump vacuum. The final product was a viscous oil. The structure was verified by ${ }^{1} \mathrm{H}$ NMR spectroscopy $\left(\mathrm{CDCl}_{3}\right): \delta=1.44$ (s, 9HC $\left.H_{3}\right), 2.13$ (s, 3HC $\left.H_{3}\right), 4.47$ (s, 12H, $\left.-\mathrm{C}\left(\mathrm{CH}_{2} \mathrm{O}-\right)_{2}\right)$, 5.85-5.89 (2d, $\left.1 \mathrm{H},-\mathrm{CH}_{2} \mathrm{CHC}(\mathrm{O}) \mathrm{O}\right), 6.08-6.18$ (dd, $\left.1 \mathrm{H},-\mathrm{CH}_{2} \mathrm{CHC}(\mathrm{O}) \mathrm{O}-\right)$, 6.40-6.46 (2d, 1H, -CH $\mathrm{CHC}(\mathrm{O}) \mathrm{O}), 6.91-7.08(\mathrm{~m}, 12 \mathrm{H},-\mathrm{OPh}-)$.

Synthesis of PolyMA-based Gels by ATRP of MA with 2A, 3A, 4A, 5A or 6A. A typical procedure for the ATRP of MA and 2A is briefly described, starting with a ratio of reagents $[\mathrm{MA}]_{0} /[2 \mathrm{~A}]_{0} /[\mathrm{EBrP}]_{0} /[\mathrm{CuBr}]_{0} /\left[\mathrm{CuBr}_{2}\right]_{0} /[\mathrm{PMDETA}]_{0}=50 / 2 / 1 / 0.45 / 0.05 / 0.5,[\mathrm{MA}]_{0}=$ 6.0 M. A clean and dry Schlenk flask was charged with MA (3.50 mL, 0.039 mol), 2A (0.290 mL, $1.56 \mathrm{mmol}$ ), PMDETA (81.2 $\mu \mathrm{L}, 0.39 \mathrm{mmol}$ ) and $N, N$-dimethylformamide (DMF, 2.51 $\mathrm{mL}$ ). The flask was deoxygenated by five freeze-pump-thaw cycles. During the final cycle, the flask was filled with nitrogen before $\mathrm{CuBr}(50.2 \mathrm{mg}, 0.35 \mathrm{mmol})$ and $\mathrm{CuBr}_{2}(8.7 \mathrm{mg}$, $0.039 \mathrm{mmol}$ ) were quickly added to the frozen mixture. No precautions were taken to avoid moisture condensation. The flask was sealed with a glass stopper then evacuated and backfilled with nitrogen five times before it was immersed in an oil bath at $60{ }^{\circ} \mathrm{C}$. Four cylindrical 
glass tubes were initially added into the reaction system. EBrP initiator $(0.101 \mathrm{~mL}, 0.78$ mmol) initiator was added to start the gelation reaction. At timed intervals, samples were withdrawn via a syringe for GC measurements of monomer conversion. The system formed a gel at certain moment when the reaction fluid lost its mobility at an upside down position for 10 seconds. After gelation, the reaction was kept at $60{ }^{\circ} \mathrm{C}$ for another two days before stopping the reaction via exposure to air. The catalyst was removed from the gel by repeated THF extraction and filtration. The experimental procedures were similar for ATRP of MA with 3A, 4A, 5A or 6A.

Synthesis of copolymer poly(HEA-co-nBA). A polymerization was conducted using reaction conditions $\quad[n \mathrm{BA}]_{0} /[\mathrm{HEA}]_{0} /[\mathrm{MBP}] /[\mathrm{CuBr}]_{0} /\left[\mathrm{CuBr}_{2}\right]_{0} /[\mathrm{PMDETA}]_{0}=$ 25/25/1/0.45/0.05/0.5 (in 25 vol\% of DMF). nBA (41.7 mmol, 6.0 mL), HEA (41.7 mol, 4.89 mL), $\mathrm{Cu}(\mathrm{II}) \mathrm{Br}_{2}$ (0.083 mmol, $\left.18.6 \mathrm{mg}\right)$, PMDETA as ligand (0.75 mmol, $0.174 \mathrm{~mL}$ ) and DMF as solvent (3.63 mL, 25 vol\%) were added to a reaction flask and the mixture was bubbled with $\mathrm{N}_{2}$ for $1 \mathrm{~h}$ to remove oxygen from the reaction mixture. After that, $\mathrm{Cu}(\mathrm{I}) \mathrm{Br}(0.749 \mathrm{mmol}$, $0.108 \mathrm{~g}$ ) was added and the reaction flask was placed in an oil bath at $50{ }^{\circ} \mathrm{C}$. When the reaction mixture reached the desired reaction temperature, the polymerization was started by adding MBP (1.66 mmol, $0.186 \mathrm{~mL}$ ) as the initiator. Samples were withdrawn periodically to monitor the monomer conversion (by GC) and the average molecular weight (by GPC). The reaction was ended after 180’ by cooling the reaction mixture in liquid nitrogen. GC analysis revealed a monomer conversion of $41 \%$ for HEA and $34 \%$ for $n \mathrm{BA}$, respectively. The copolymer was separated from the monomer by precipitation in a large excess of cold distilled water, followed by centrifugation. After drying in vacuum at room temperature overnight, the pure copolymer was obtained as a highly viscous material.

Synthesis of multivinyl cross-linker based on poly(HEA-co-nBA). Poly(HEA-co$n$ BA) copolymer $\left(M_{n}=1700 \mathrm{~g} / \mathrm{mol}\right.$ and $\left.\mathrm{M}_{\mathrm{w}} / \mathrm{M}_{\mathrm{n}}=1.11\right)$ was reacted with acryloyl chloride in 
a similar procedure as described above. $0.1 \mathrm{~mol} \%$ of radical inhibitor 4-hydroxy-TEMPO was added relative to vinyl groups to prevent cross-linking during the esterification reaction. The reaction product was purified by dialysis in THF, yielding a polymer with pendant vinyl groups $\left(M_{n}=1850 \mathrm{~g} / \mathrm{mol}\right.$ and $\left.M_{w} / M_{n}=1.12\right) .{ }^{1} \mathrm{H}$ NMR spectroscopy of the pure polymer revealed an esterification yield of about $90 \%$.

\section{Results and Discussion}

The ATRP of MA monomer and various cross-linkers containing different numbers of acrylate moieties was performed using $\mathrm{CuBr} / \mathrm{PMDETA}$ as the catalyst $\left(10 \mathrm{~mol} \%\right.$ of $\mathrm{CuBr}_{2}$ of total copper species was pre-added in the system), ethyl-2-bromopropionate (EBrP) as the initiator with an initial molar ratio of $[\mathrm{MA}]_{0} /[\mathrm{EBrP}]_{0}=50$ in $\mathrm{DMF}\left([\mathrm{MA}]_{0}=6.0 \mathrm{M}\right)$ at $60{ }^{\circ} \mathrm{C}$. These reaction conditions have been previously reported as a good system for ATRP of acrylate-based monomers, exhibiting fast initiation kinetics, a high initiation efficiency, and controlled polymer chain growth with low polydispersity of the primary chains. ${ }^{14}$

In this paper, two approaches were employed to investigate the effect of cross-linker multiplicity on experimental gelation. First, the concentration of branching vinyl groups (molar ratio of $\left[\mathrm{Vinyl}_{\text {branch }}\right]_{0} /[\text { Initiator }]_{0}$ ) was kept constant for series of reactions with increasing cross-linker multiplicity. Additionally, the effect of cross-linker multiplicity was investigated in a series of reactions where the molar concentration of the various cross-linkers was kept at a constant value.

\section{Gelation reactions with constant molar ratio of [Vinyl $\left.{ }_{\text {branch }}\right]_{0} /[\text { Initiator }]_{0}$.}

In the first series of reactions, gelation reactions with ethylene glycol diacrylate (2A) and trimethylolpropane triacrylate (3A) cross-linkers were compared. The molar ratio of vinyl groups that can introduce branching ( 2 per molecule for $2 \mathrm{~A} ; 3$ for $3 \mathrm{~A}$, etc.) to initiator was 
kept at a constant value (constant $\left.\left[\mathrm{Vinyl}_{\mathrm{branch}}\right]_{0} /[\text { Initiator }]_{0}\right)$. For various molar ratios of [Vinyl $\left.\mathrm{branch}_{0}\right]_{0} /[\text { Initiator }]_{0}$ ranging from 2.20 to 10 , experimental gelation occurred consistently at lower MA conversions for reactions with the trivinyl cross-linker 3A, as compared to the use of divinyl cross-linker (Table 1). Upon incorporation of a trivinyl cross-linker into a growing polymer chain, two pendant vinyl groups are generated. If one of them is consumed by intramolecular cyclization, the second one is still available to react and form an intermolecular cross-linkage. In contrast, if the pendant vinyl group from an incorporated divinyl cross-linker reacts intramolecularly, this cross-linker does not contribute to the increase of the molecular weight of the branched polymers. As a result, the use of a trivinyl cross-linker for the copolymerization leads to a network with a higher extent of intermolecular cross-linkages than the use of 1.5 eq. of divinyl cross-linker.

In similar series of reactions with various molar ratios of $\left[\mathrm{Viny}_{\text {branch }}\right]_{0} /[\text { Initiator }]_{0}$, gelation reactions were also conducted using a tetravinyl cross-linker (pentaerythritol tetraacrylate, 4A), a pentavinyl cross-linker (dipentaerythritol pentaacrylate, 5A) and a hexafunctional acrylate cross-linker (6A). It should be noted that 5A cross-linker is based on dipentaerythritol penta-/hexa-acrylate, which could contain small amounts of triester and hexafunctional compound. In our study, no further purification was performed as the average multiplicity was determined by ${ }^{1} \mathrm{H}$ NMR as being a pentafunctional acrylate. The synthesis of hexafunctional acrylate 6A is depicted in Scheme 2. The results of the gelation reactions are summarized in Table 1. 


\section{Table 1. Comparison of ATRP reactions of MA using cross-linkers with different multiplicity. ${ }^{1}$}

\begin{tabular}{llllll}
\hline [Vinyl $\left._{\text {branch }}\right]_{0}$ & \multicolumn{2}{l}{ Conv $_{\text {MA,gel }}{ }^{3}$} & & & \\
\cline { 2 - 6 }$/[\text { Initiator }]_{0}$ & $2 \mathrm{~A}$ & $3 \mathrm{~A}$ & $4 \mathrm{~A}$ & $5 \mathrm{~A}$ & $6 \mathrm{~A}$ \\
\hline 2.20 & 0.97 & 0.94 & 0.89 & 0.99 & $/^{4}$ \\
3.00 & 0.86 & 0.78 & 0.79 & 0.83 & $/^{4}$ \\
4.00 & 0.79 & 0.67 & 0.67 & 0.65 & 0.82 \\
10.0 & 0.48 & 0.40 & 0.39 & 0.34 & 0.34
\end{tabular}

1. Experimental conditions: 1) "2A" reaction: ATRP of MA and 2A with $\left.[\mathrm{MA}]_{0} /[2 \mathrm{~A}]_{0} /[\mathrm{EBrP}]_{0} /[\mathrm{CuBr}]_{0} /\left[\mathrm{CuBr}_{2}\right]_{0} /[\mathrm{PMDETA}]_{0}=50 / \mathrm{A} / 1 / 0.45 / 0.05 / 0.5 ; 2\right)$ "3A" reaction: ATRP of MA and 3A: $\left.[\mathrm{MA}]_{0} /[3 \mathrm{AA}]_{0} /[\mathrm{EBrP}]_{0} /[\mathrm{CuBr}]_{0} /\left[\mathrm{CuBr}_{2}\right]_{0} /[\mathrm{PMDETA}]_{0}=50 / \mathrm{B} / 1 / 0.45 / 0.05 / 0.5 .3\right)$ ATRP of MA and 4A: $[\mathrm{MA}]_{0} /[3 \mathrm{~A}]_{0} /[\mathrm{EBrP}]_{0} /[\mathrm{CuBr}]_{0} /\left[\mathrm{CuBr}_{2}\right]_{0} /[\mathrm{PMDETA}]_{0}=50 / \mathrm{C} / 1 / 0.45 / 0.05 / 0.5$. 4) ATRP of MA and 5A: $\left.[\mathrm{MA}]_{0} /[5 \mathrm{~A}]_{0} /[\mathrm{EBrP}]_{0} /[\mathrm{CuBr}]_{0} /\left[\mathrm{CuBr}_{2}\right]_{0} /[\mathrm{PMDETA}]_{0}=50 / \mathrm{D} / 1 / 0.45 / 0.05 / 0.5 .5\right)$ ATRP of MA and $6 \mathrm{~A}$ : $[\mathrm{MA}]_{0} /[6 \mathrm{~A}]_{0} /[\mathrm{EBrP}]_{0} /[\mathrm{CuBr}]_{0} /\left[\mathrm{CuBr}_{2}\right]_{0} /[\mathrm{PMDETA}]_{0}=50 / \mathrm{E} / 1 / 0.45 / 0.05 / 0.5$. All reactions were performed with $[\mathrm{MA}]_{0}=6.0 \mathrm{M}$ in DMF at $60{ }^{\circ} \mathrm{C}$ and stopped after 2 days in order to reach the complete conversions.

2. $\left[\text { Vinyl } \mathrm{branch}_{0}\right]_{0} /[\text { Initiator }]_{0}=(2 \times \mathrm{A})$ for " $2 \mathrm{~A}$ " reaction, $(3 \times \mathrm{B})$ for " $3 \mathrm{~A}$ " reaction, $(4 \times \mathrm{C})$ for reaction with $4 \mathrm{~A}$, $(5 \times D)$ for reaction with $5 \mathrm{~A}$, and $(6 \times \mathrm{E})$ for reaction with $6 \mathrm{~A}$. For the comparable reactions with the same value of $\left[V_{\text {Viny }} \mathrm{b}_{\text {branch }}\right]_{0} /[\text { Initiator }]_{0}, \mathrm{~B}=2 \times \mathrm{A} / 3, \mathrm{C}=\mathrm{A} / 2, \mathrm{D}=2 \times \mathrm{A} / 5, \mathrm{E}=\mathrm{A} / 3$.

3. Conversions of MA immediately before gelation. The experimental gel point was the moment when the reaction fluid lost its mobility when held at an upside down position for 10 seconds.

4. No gelation occurred under these conditions. 


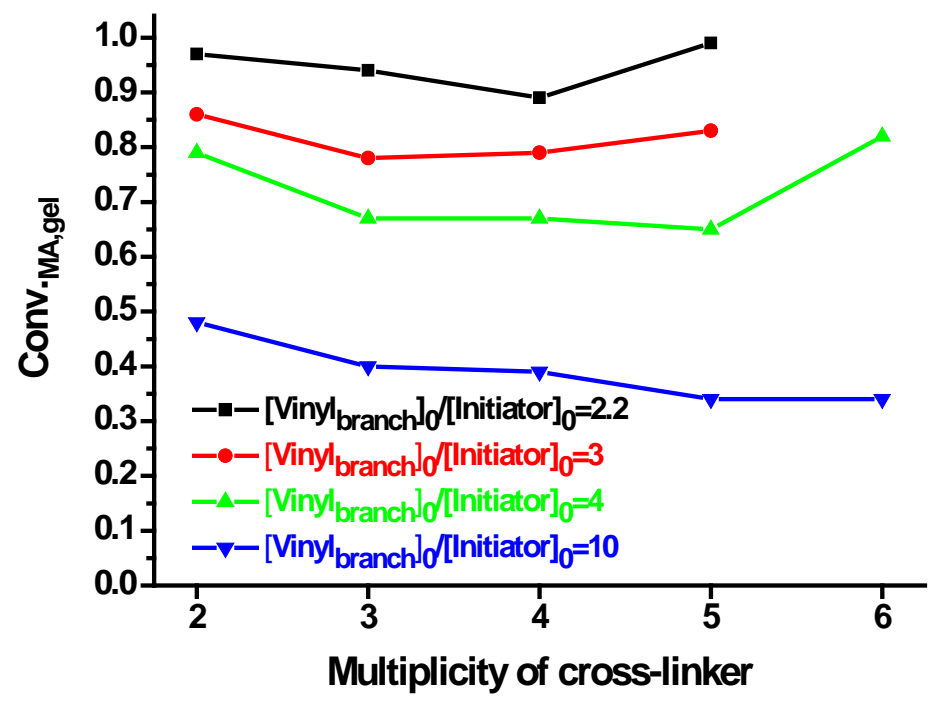

Figure 1. Conversion of MA at gelation for ATRP of MA and cross-linker as a function of cross-linker multiplicity for various constant molar ratios of $\left[\mathrm{Vinyl}_{\text {branch }}\right]_{0} /[\text { Initiator }]_{0}$.

For reactions using the trivinyl cross-linker 3A, gelation occurred at consistently lower monomer conversion as compared to the gelation by using 2A cross-linker (Figure 1). However, for reactions using the tetravinyl cross-linker 4A, no significant influence of the cross-linker multiplicity was observed under the same $\left[\text { Vinyl }_{\text {branch }}\right]_{0} /[\text { Initiator }]_{0}$ ratio, as compared to the gelation by using 3A cross-linker. When the cross-linker containing five acrylate moieties per molecule was used, a different trend was observed in comparable reactions. For low [Vinyl $\left.\mathrm{branch}_{0}\right]_{0} /[\text { Initiator }]_{0}$ ratios (i.e. 2.2 and 3), gelation seems to be delayed with increasing multiplicity of the cross-linker. For instance, for the reaction with 5A, the experimental gel point was only observed at $99 \%$ conversion for a ratio of $\left[\text { Vinyl }_{\text {branch }}\right]_{0} /[\text { Initiator }]_{0}$ ratio $=2$ 2.2. In contrast, for higher $\left[\text { Vinyl } l_{\text {branch }}\right]_{0} /[\text { Initiator }]_{0}$ ratios (i.e. 4 and 10), increased multiplicity of the cross-linker still resulted in gelation at earlier MA conversion. The effect tends to be even more clear for reactions with $\left[\text { Vinyl }{ }_{\text {branch }}\right]_{0} /[\text { Initiator }]_{0}$ $=10$. This trend is confirmed for the reactions using hexafunctional cross-linker 6A. In this 
case, no gelation was observed for reactions with a $\left[\text { Vinyl } l_{\text {branch }}\right]_{0} /[\text { Initiator }]_{0}$ ratio $=2.2$ and 3 , while gelation was significantly delayed for the reaction with a $\left[\mathrm{Vinyl}_{\text {branch }}\right]_{0} /[\text { Initiator }]_{0}$ ratio $=4$. Only for the reaction with a $\left[\operatorname{Vinyl}_{\text {branch }}\right]_{0} /[\text { Initiator }]_{0}$ ratio $=10$, no gelation delay was observed. We attribute these observations to a dilution effect: a constant $\left[\mathrm{Vinyl}_{\text {branch }}\right]_{0} /[\text { Initiator }]_{0}$ ratio directly implies that the concentration of cross-linker in the reaction medium decreases with increasing multiplicity of the cross-linker. This is schematically depicted in Figure 2.

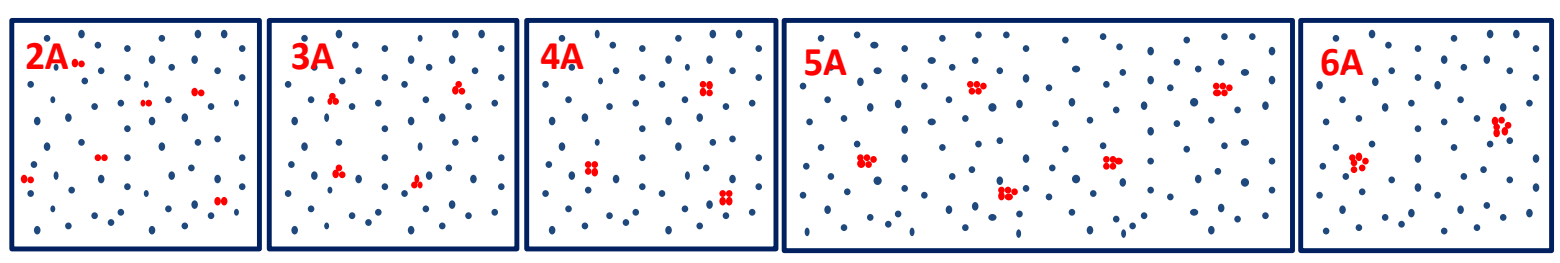

Figure 2. Schematic depiction of reaction medium of ATRP of MA (blue dots) and various cross-linkers with different multiplicity (in red: 2A, 3A, 4A, 5A and 6A containing 2, 3, 4, 5, or 6 vinyl groups per molecule, respectively) for a series of gelation reactions with constant molar ratio of $\left[\text { Vinyl }_{\text {branch }}\right]_{0} /[\text { Initiator }]_{0}=12$.

For low $\left[\text { Vinyl }_{\text {branch }}\right]_{0} /[\text { Initiator }]_{0}$, the cross-linker concentration may drop below a critical value, resulting in a more pronounced intramolecular cyclization as the effect of a lower concentration in the reaction medium. It was previously reported that a decreasing monomer concentration results in delayed experimental gelation due to significantly increased intramolecular cyclization,. ${ }^{15}$ Similarly, a decreased cross-linker concentration, originating from the increased multiplicity of the cross-linker with a constant ratio of $\left[\text { Vinyl }_{\text {branch }}\right]_{0} /[\text { Initiator }]_{0}$, could also lead to delayed experimental gelation. 
On the other hand, if the $\left[\mathrm{Vinyl}_{\mathrm{branch}}\right]_{0} /[\text { Initiator }]_{0}$ ratio is rather high, the cross-linker concentration may be sufficient to prevent an intramolecular cyclization, resulting in earlier gelation with increasing cross-linker multiplicity.

\section{Gelation reactions with constant molar concentration of cross-linker.}

The previously discussed reactions with a constant $\left[\mathrm{Vinyl}_{\text {branch }}\right]_{0} /[\text { Initiator }]_{0}$ ratio correlated the experimental gelation behavior with a dilution effect, resulting from an increasing multiplicity of the cross-linker. However, gelation reactions with a relatively high $\left[\text { Vinyl }_{\text {branch }}\right]_{0} /[\text { Initiator }]_{0}$ ratio still showed gelation at lower monomer conversion for increasing multiplicity of the cross-linker. To further investigate the influence of the crosslinker multiplicity in relation with its concentration, two series of gelation reactions were performed with two constant molar ratios of [monomer]/[initiator]/[cross-linker] $\left([\mathrm{M}]_{0} /[\mathrm{I}]_{0} /[\mathrm{X}]_{0}\right)=50 / 1 / 1$ and 50/1/2, under similar reaction conditions as described above. In other words, the molar concentration of the cross-linker in the medium is identical for all gelation reactions, regardless of the number of branching vinyl groups per cross-linker molecule (see schematic depiction in Figure 3).

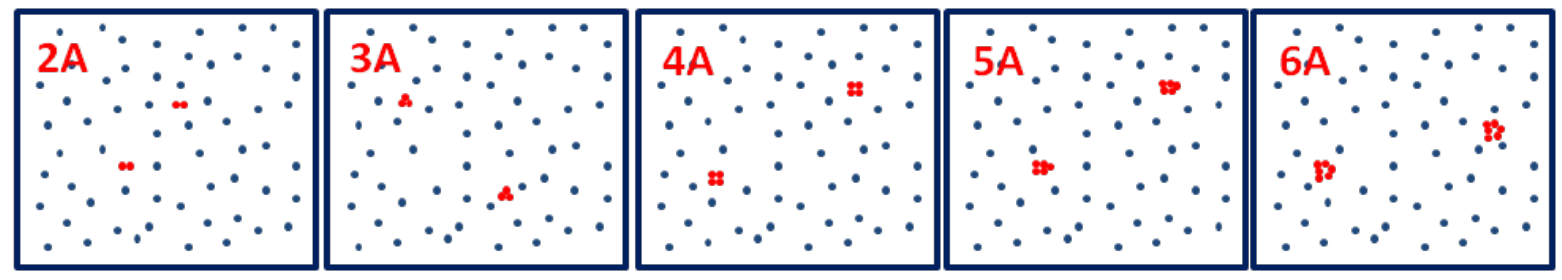

Figure 3. Schematic depiction of reaction medium of ATRP of MA (blue dots) and various cross-linkers with different multiplicity (in red: 2A, 3A, 4A, 5A and 6A containing 2, 3, 4, 5, or 6 vinyl groups per cross-linker molecule, respectively) for a series of gelation reactions with a constant concentration of cross-linker. 
In contrast to reactions with a constant molar ratio of $\left[\mathrm{Vinyl}_{\text {branch }}\right]_{0} /[\text { Initiator }]_{0}$ for the various cross-linkers, this study avoids the influence of effects that are directly related to dilution of the cross-linker molecules in the reaction medium. For example, for the series of reactions with $[\mathrm{M}]_{0} /[\mathrm{I}]_{0} /[\mathrm{X}]_{0}=50 / 1 / 1$, the $\left[\operatorname{Vinyl}_{\text {branch }}\right]_{0} /[\text { Initiator }]_{0}$ ratio was $2,3,4,5$ and 6 , for divinyl cross-linker 2A, trivinyl cross-linker 3A, tetravinyl cross-linker 4A, pentavinyl cross-linker $5 \mathrm{~A}$ and hexavinyl cross-linker $6 \mathrm{~A}$, respectively. At $\left[\mathrm{Viny}_{\mathrm{branch}}\right]_{0} /[\text { Initiator }]_{0}=2$, no gelation was observed when 2A was used as cross-linker. The experimental gel points for these series of gelation reactions with constant concentration of cross-linker are displayed in Figure 4. For a constant concentration of the cross-linker, gelation occurred consistently at lower MA conversions for reactions with an increasing number of vinyl groups per cross-linker molecule. A similar trend was observed for a series of gelation reactions with $[\mathrm{M}]_{0} /[\mathrm{I}]_{0} /[\mathrm{X}]_{0}=$ 50/1/2.

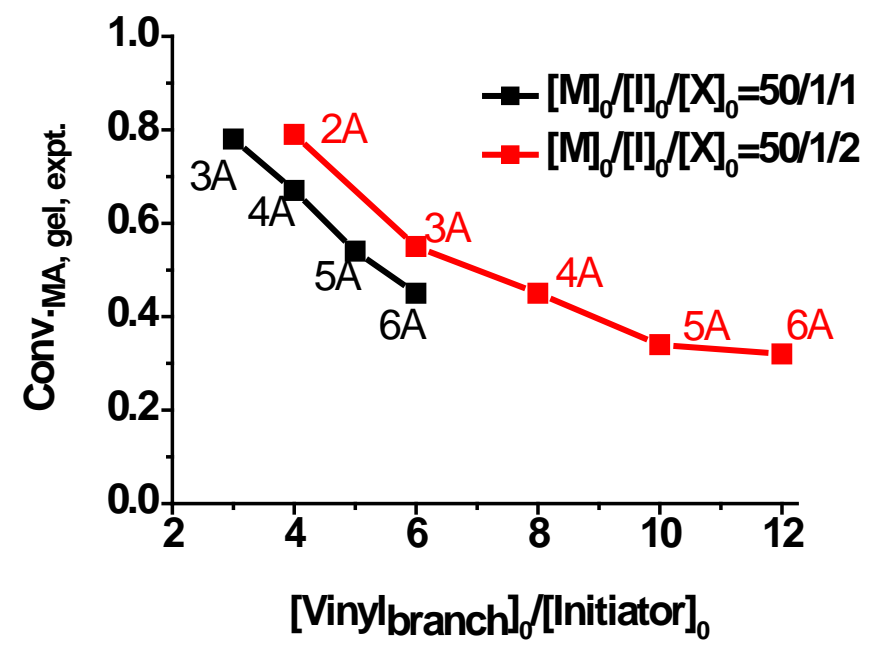

Figure 4. Conversion of MA at experimental gel point for ATRP of MA and cross-linker with various multiplicities, using a constant molar cross-linker concentration [X]. 


\section{Determination of pendant vinyl groups in the sols.}

The reactions with various constant ratios of [Vinyl $\left.\mathrm{branch}_{0}\right]_{0} /[\text { Initiator }]_{0}$ for various cross-linkers with different multiplicity suggested a gelation behavior related to the concentration of the cross-linker in the reaction medium. To further investigate this effect, the number of unreacted pendant vinyl groups in the sols was determined for reactions with different multiplicity of the cross-linker and various ratios of $\left[\mathrm{Vinyl}_{\text {branch }}\right]_{0} /[\text { Initiator }]_{0}$. If equal reactivity of each vinyl group is assumed, the fraction of each species originating from the multivinyl cross-linker is determined by the statistical combination formula below.

$$
\begin{aligned}
& P(K=k)=\left(\bar{\Psi}_{k}^{n}\right) p^{k}(1-p)^{n-k} \\
& \text { where }\left(\bar{\Psi}_{k}^{n}\right)=\frac{n !}{k !(n-k) !}
\end{aligned}
$$

$\mathrm{p}$ is conversion of $\mathrm{M}$

and $\mathrm{k}=$ number of vinyl groups reacted out of total number $\mathrm{n}$.

This formula expresses the probability that $k$ (out of total number $n$ ) vinyl groups have reacted at monomer conversion p, and defines the fraction of each species relative to the starting amount of unreacted cross-linker. For a cross-linker containing n vinyl groups, $n+1$ species can be distinguished. For example, 4 different species originating from the cross-linker can be observed in the ATRP of monomer and 3A cross-linker: (i) a fraction with none of the vinyl groups reacted $(\mathrm{k}=0)$, representing the unreacted free cross-linker; (ii) a fraction of the crosslinker with one vinyl group reacted $(k=1)$, which is incorporated in a primary chain and contains 2 pendant vinyl groups; (iii) a fraction of the cross-linker with two vinyl groups reacted $(\mathrm{k}=2)$ and 1 pendant vinyl group; and (iv) a fraction of cross-linker that has fully reacted $(\mathrm{k}=3)$. Therefore, the theoretical fraction of the pendant vinyl groups in the sols $\left(f_{\mathrm{A}, \mathrm{p}}\right)$ during ATRP of monomer and trivinyl cross-linker is the sum of the pendant vinyl groups present in the fractions of $\mathrm{k}=1$ and $\mathrm{k}=2$. 
The theoretical and experimental fractions of pendant vinyl groups in the sols $\left(f_{\mathrm{A}, \mathrm{p}}\right)$ as a function of MA conversion during ATRP of MA and pentavinyl cross-linker 5A were compared for a few reactions with various molar ratios of $\left[\text { Vinyl }_{\text {branch }}\right]_{0} /[\text { Initiator }]_{0}$ (Figure 5). The dots in Figure 5 represent the experimental results of $f_{\mathrm{A}, \mathrm{p}}$ determined by ${ }^{1} \mathrm{H}$ NMR analysis of the sols just before gelation. Unreacted MA and free cross-linker were removed from the polymer samples by repeated precipitation/redissolving in hexane/tetrahydrofuran. ${ }^{1} \mathrm{H}$ NMR integration results determined the molar ratio of pendant vinyl groups to reacted MA units in the polymer backbone, which was further converted into the $f_{\mathrm{A}, \mathrm{p}}$ taking into account the MA conversion. ${ }^{14}$ The results in Figure 5 show that the experimental $f_{\mathrm{A}, \mathrm{p}}$ was in good agreement with the calculated $f_{\mathrm{A}, \mathrm{p}}$ for reactions with relatively low ratio of $\left[\mathrm{Vinyl}_{\mathrm{branch}}\right]_{0} /[\text { Initiator }]_{0}$ (i.c. 3 and 4). However, the experimental value of $f_{\mathrm{A}, \mathrm{p}}$ was higher than the theoretical value when the ratio of $\left[\mathrm{Viny}_{\mathrm{branch}}\right]_{0} /[\text { Initiator }]_{0}$ was 10 . This difference could be attributed to the steric protection of dangling chains, resulting in the pendant vinyl groups less likely to react. For a relatively low [Vinyl $\left.\mathrm{branch}_{0}\right]_{0} /[\text { Initiator }]_{0}$ ratio (3 and 4), the distance between the various crosslinkages would be relatively larger (lower cross-link density), compared to reactions with a higher $\left[\text { Vinyl }_{\text {branch }}\right]_{0} /[\text { Initiator }]_{0}$ ratio (6 and 10; higher cross-link density). Intermolecular cross-linking reactions would be more difficult in the latter case, resulting in a higher $f_{\mathrm{A}, \mathrm{p}}$ than the theoretical value.

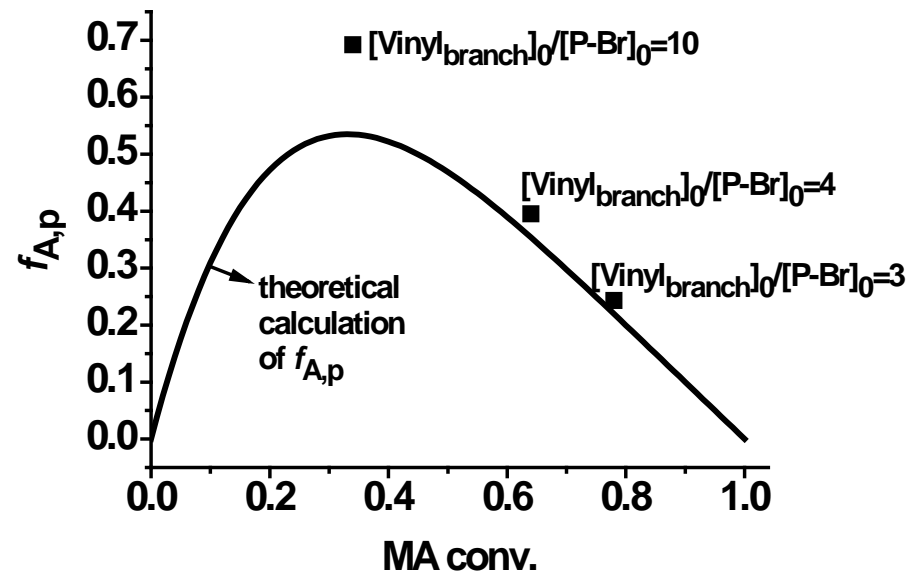


Figure 5. Comparison of calculated results (solid line) and experimental results (dots) of the fraction of pendant acrylate groups $\left(f_{\mathrm{A}, \mathrm{p}}\right)$ as a function of MA conversion during the ATRP of MA and pentaacrylate cross-linker 5A.

Further investigation for a molar ratio of $\left[\mathrm{Vinyl}_{\text {branch }}\right]_{0} /[\text { Initiator }]_{0}=10$ was done for trivinyl cross-linker 3A, and tetravinyl cross-linker 4A. It was previously reported that theoretical and experimental $f_{\mathrm{A}, \mathrm{p}}$ values for ATRP of MA and 2A were in a good agreement. ${ }^{14,26}$ For relatively low $\left[\mathrm{Vinyl}_{\text {branch }}\right]_{0} /[\text { Initiator }]_{0}$ ratio (i.c. 3 and 4), no significant differences between the theoretical and experimental $f_{\mathrm{A}, \mathrm{p}}$ for ATRP of MA and 3A and 4A were observed, just as in the case of ATRP of MA and 5A. However, for a high ratio of [Vinyl $\left.{ }_{\text {branch }}\right]_{0} /[\text { Initiator }]_{0}$ (i.c. 10), a growing discrepancy between the theoretical and experimental $f_{\mathrm{A}, \mathrm{p}}$ was found with increasing multiplicity of the cross-linker (see Figure 6).

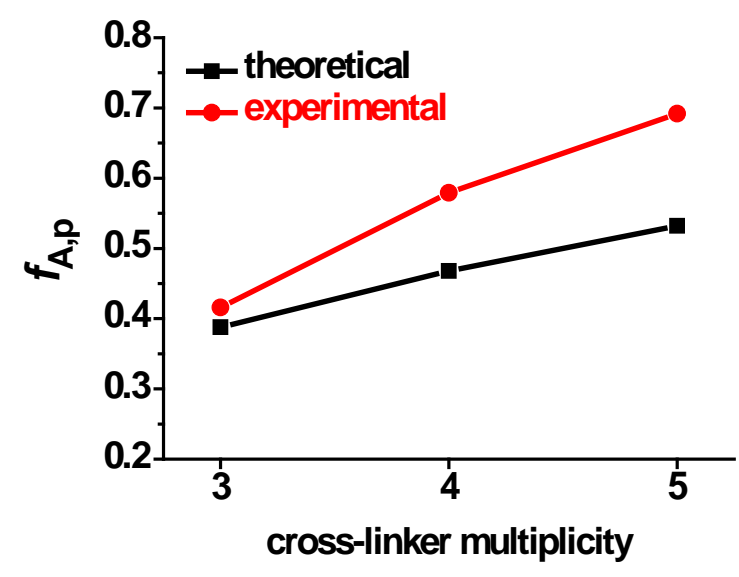

Figure 6. Theoretical and experimental fraction of pendant acrylate groups $\left(f_{\mathrm{A}, \mathrm{p}}\right)$ in the sols as a function of cross-linker multiplicity for ATRP of MA and 3A, 4A and 5A, respectively, for $\left[\text { Vinyl }_{\text {branch }}\right]_{0} /[\text { Initiator }]_{0}$ ratio $=10$. Samples were taken close to the gelation point.

\section{Gelation with a polymeric multivinyl cross-linker (7A).}


In addition to the use of the cross-linkers with well-defined number of vinyl groups per molecule, we have also studied a gelation in the presence of an oligomeric (polymer-based) cross-linker. The cross-linker was prepared based on a random copolymer of $n$-butyl acrylate (nBA) and 2-hydroxyethyl acrylate (HEA), followed by esterification of the hydroxyl groups in the side chain to acrylate moieties using acryloyl chloride. The average number of acrylate moieties per macromolecule was 7 , as determined by ${ }^{1} \mathrm{H}$ NMR. In the reactions with a constant molar ratio of $\left[\text { Vinyl }_{\text {branch }}\right]_{0} /[\text { Initiator }]_{0}=4$, the ATRP of MA and multivinyl crosslinker 7A showed no gelation up to $91 \%$ MA conversion, in contrast to reactions with 5A (experimental gelation at $65 \%$ MA conversion) and 6A (experimental gelation at $82 \% \mathrm{MA}$ conversion). In the reactions with a constant concentration of cross-linker $\left([\mathrm{M}]_{0} /[\mathrm{I}]_{0} /[\mathrm{X}]_{0}=\right.$ 50/1/1, ATRP of MA and 7A showed a delayed gelation (at 99\% MA conversion) in comparison to a similar reaction with the same concentration of 5A (gelation at 54\% MA conversion). The difference in the gelation behavior could be attributed to the oligomeric nature of the multivinyl cross-linker and also to the linear structure of 7A. The acrylate crosslinking groups located next to each other along the backbone could facilitate intramolecular cross-linking reactions.

\section{Conclusion}

The effect of various types of cross-linkers containing different numbers of vinyl groups per molecule (2, 3, 4, 5 or 6 ) on the gelation behavior was studied during the ATRP of MA. At a constant ratio of branching vinyl groups to initiator, a concentration-dependant gelation for cross-linkers with different numbers of vinyl groups per molecule was observed. At a low targeted cross-link density, the experimental gelation was delayed for cross-linkers with higher number of vinyl groups per molecule (5 or 6). At a higher targeted cross-link density, 
increasing the number of vinyl groups per cross-linker molecule led to slightly accelerated gelation. When a constant molar concentration of various cross-linkers was used, experimental gelation occurred consistently at lower monomer conversions for reactions with a higher number of vinyl groups per cross-linker molecule. Moreover, the amount of unreacted pendant vinyl groups in the sols was determined for a series of reactions with various cross-linkers and different molar ratios of $\left[\text { Vinyl }_{\text {branch }}\right]_{0} /[\text { Initiator }]_{0}$. The experimentally determined fraction of pendant acrylate groups $\left(f_{\mathrm{A}, \mathrm{p}}\right)$ was significantly higher than the value theoretically predicted for gelation at a high targeted cross-link density. The

discrepancy between the theoretical and experimental $f_{\mathrm{A}, \mathrm{p}}$ increased with increasing number of vinyl groups per cross-linker molecule $(5 \mathrm{~A}>4 \mathrm{~A}>3 \mathrm{~A})$. These results help to better understand the gelation process in controlled radical copolymerization of monomer and crosslinkers with variable multiplicities.

\section{Acknowledgement}

W. Van Camp thanks the Research Foundation - Flanders (FWO) for a postdoctoral fellowship and mobility allowance. F. Du Prez is grateful to the Belgian Program on Interuniversity Attraction Poles initiated by the Belgian State, Prime Minister's office (Program P6/27) for financial support. The financial support from NSF (DMR-05-49353) and the CRP Consortium at Carnegie Mellon University is also greatly appreciated.

\section{References}

$1 \quad$ Landin, D. T.; Macosko, C. W. Macromolecules 1988, 21, 846-851.

2 Zhu, S.; Tian, Y.; Hamielec, A. E.; Eaton, D. R. Polymer 1990, 31, 154-159.

3 Kannurpatti, A. R.; Anseth, J. W.; Bowman, C. N. Polymer 1998, 39, 2507-2513.

4 Bastide, J.; Leibler, L. Macromolecules 1988, 21, 2647-2649. 
Braunecker, W. A.; Matyjaszewski, K. Prog. Polym. Sci. 2007, 32, 93-146.

6 Matyjaszewski, K.; Xia, J. H. Chem. Rev. 2001, 101, 2921-2990.

7 Kamigaito, M.; Ando, T.; Sawamoto, M. Chem. Rev. 2001, 101, 3689-3745.

8 Tsarevsky, N. V.; Matyjaszewski, K. Chem. Rev. 2007, 107, 2270-2299.

9 Matyjaszewski, K.; Tsarevsky, N. V. Nature Chem. 2009, 1, 276-288.

10 Gao, H.; Matyjaszewski, K. Progr. Polym. Sci. 2009, 34, 317-350.

11 Ide, N.; Fukuda, T. Macromolecules 1999, 32, 95-99.

12 Oh, J. K.; Drumright, R.; Siegwart, D. J.; Matyjaszewski, K. Prog. Polym. Sci. 2008, 33, 448-477.

13 Yu, Q.; Zeng, F. Q.; Zhu, S. P. Macromolecules 2001, 34, 1612-1618.

14 Gao, H.; Min, K.; Matyjaszewski, K. Macromolecules 2007, 40, 7763-7770.

15 Gao, H.; Li, W.; Matyjaszewski, K. Macromolecules 2008, 41, 2335-2340.

16 Gao, H.; Miasnikova, A.; Matyjaszewski, K. Macromolecules 2008, 41, 7843-7849.

17 Li, W.; Gao, H.; Matyjaszewski, K. Macromolecules 2009, 42, 927-932.

18 Bannister, I.; Billingham, N. C.; Armes, S. P. Soft Matter 2009, 5, 3495-3504.

19 Bannister, I.; Billingham, N. C.; Armes, S. P.; Rannard, S. P.; Findlay, P. Macromolecules 2006, 39, 7483-7492.

20 Bouhier, M. H.; Cormack, P. A. G.; Graham, S.; Sherrington, D. C. J. Polym. Sci. Part A: Polym. Chem. 2007, 45, 2375-2386.

21 Isaure, F.; Cormack, P. A. G.; Graham, S.; Sherrington, D. C.; Armes, S. P.; Butun, V. Chem. Commun. 2004, 1138-1139.

22 Li, Y. T.; Armes, S. P. Macromolecules 2005, 38, 8155-8162.

23 Wang, A. R.; Zhu, S. P. Polym. Eng. Sci. 2005, 45, 720-727.

24 Wang, A. R.; Zhu, S. P. J. Polym. Sci. Part A: Polym. Chem. 2005, 43, 5710-5714.

25 Yu, Q.; Zhang, J. Z.; Cheng, M. L.; Zhu, S. P. Macromol. Chem. Phys. 2006, 207, 287-294.

26 Gao, H. F.; Polanowski, P.; Matyjaszewski, K. Macromolecules 2009, 42, 5925-5932.

27 Gao, H. F.; Min, K.; Matyjaszewski, K. Macromolecules 2009, 42, 8039-8043.

28 Gao, H.; Tsarevsky, N. V.; Matyjaszewski, K. Macromolecules 2005, 38, 5995-6004. 